\title{
DAS VOLUMGEWICHT ALS KENNZEICHEN DER "NORMALEN" BODENSTRUKTUR
}

\author{
Reijo Heinonen \\ Agrikulturchemisches Institut der Universität Helsinki \\ Einleitung
}

Eingegangen 18. 1. 1960

Häufig erwartet man von der Bodenphysik eine Antwort auf die Frage, ob die schlechte Struktur des Bodens, in erster Linie seine übermässige Dichtigkeit die Ursache des festgestellten schlechten Wuchses ist. Vor einen solchen Fall gestellt, strebt ein Forscher im allgemeinen als Erstes die Hohlraumverhältnisse im Profil zu ergründen. Indessen muss man sehr grosse Vorsicht beobachten, ehe man auf Grund solcher Messergebnisse einen Boden als "schlecht» bezeichnet, denn anscheinend sogar sehr «;chlechte Struktur» aufweisende Böden können bei geschickter Behandlung dauernd gute Erträge liefern (1). Im Ermitteln der Gründe für das schlechte Wachstum hat man einen Schritt vorwärts getan, wenn man wenigstens richtunggebend są̧en kann, ob der Boden höhere Dichtigkeit als normal besitzt. Als Vergleichsbasis würde man dabei die hypothetische mormalen Dichtigkeit der in Frage stehenden Bodenart, entweder durch die Gesamtporosität oder durch das Volumgewicht ausફ,edrückt, benötigen.

Das Volumgewicht ist recht allgemein als eine Kennzahl der Bodenstruktur angewandt worderı. Es hat unter anderem den Vorteil einer verhältnismässig raschen und genauen Bestimmbarkeit und, infolge des absoluten Charakters der Werte, einer guten Vergleichbarkeit. Vomocil (9) hat kürzlich eine Übersicht der Verfahren zur Bestimmung des Volumgewichts sowie seiner Verwertung in bodenphysikalischen Untersuchungen veröffentlicht.

\section{Das Volumgewicht der Ackerkrume}

Die vorliegende Arbeit gilt dem Versuch, das normale Volumgewicht der Ackerkrume der bebauten glazialen Böden in Süd-Finnland in Abhängigkeit von der Textur und dem Humusgehalt des Bodens zu ermitteln, wozu ein aus 111 Proben 


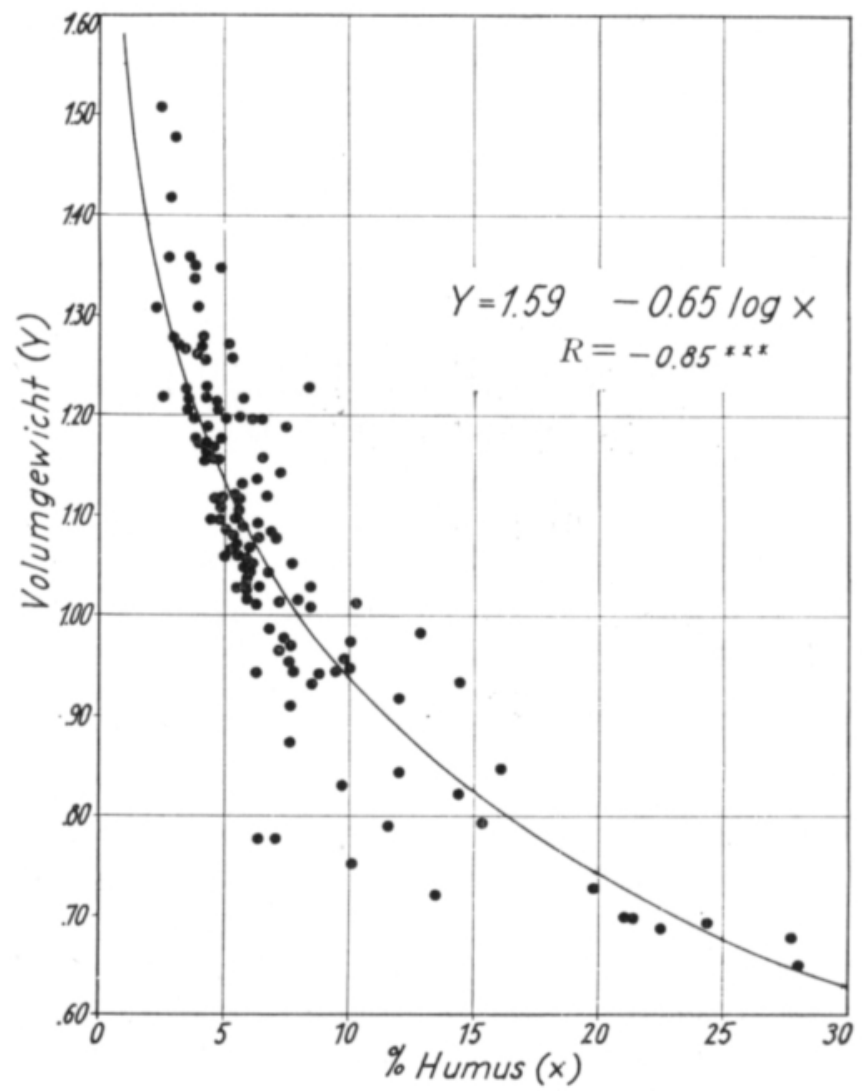

Abb. 1. Das Volumgewicht als Funktion des Humusgehaltes im Boden.

bestehendes Material herangezogen wurde. Die Proben sind mit einem Zylinder 2 bis 4 Jahre alten Wiesen in 5 bis $15 \mathrm{~cm}$ Tiefe entnommen worden, und zwar befand sich der Boden hierbei jeweils im Feldkapazitätszustand. Das so ermittelte Volumgewicht ist ein exakt definierter Begriff und eignet sich in den finnischen Verhältnissen gut dazu, die von Faktoren zufälliger Art freie "normale» Struktur des Bodens zu vertreten. Der Verfasser hat zuvor in verschiedenen Publikationen $(2,3,6)$ über die physikalischen und chemischen Eigenschaften des gleichen Probenmaterials berichtet.

Abb. 1 gibt die Abhängigkeit des Volumgewichts vom Humusgehalt (Humus = $1,7 \mathrm{x}$ org. C) wieder. Die Beziehung ist krummlinig; sie kann indessen mittels einer einfachen Regressionsgleichung wiedergegeben werden, indem man den Humusgehalt im logarithmischen Mass ausdrückt (2, S. 45). Der mit einem zweifelhaften Koeffizienten berechnete "Humusgehalt" kann auch durch den exakteren Beriff morganischer Totalkohlenstoff» ersetzt werden, dessen Bestimmung hier auf einer nachträglich durchgeführten Elementaranalyse der Vergleichsbodenprobe begründet ist (7). Die in der Abb. 1 wiedergegebene Gleichung erhält dann folgende Form:

$$
\text { Volumgewicht }=1.47-0.65 \log (\text { org. total-C) }
$$


In der vorliegenden Arbeit wurde der Teil des Materials mit weniger als $12 \%$ Humusgehalt einer riäheren Betrachtung unterzogen. In diesem Bereich ist in Abb. 1 eine beträchtlich hohe Streuung zu beobachten und in einem so engen Variationsintervall kann man keine Behauptungen betreffs einer nichtlinearen Beziehung aufstellen. Vorbereitende Betrachtungen zeigten, dass das Volumgewicht ausser vom Humusgehalt c.uch von der Textur abhängt, indem Ton zur Eriniedrigung und Sand zur Erhöhung desselben beiträgt. Die Tonfraktion $(<2 \mu)$ variiert in dem vorliegenden Material in den Grenzen 2-75\% und die Sandfraktion $(>60 \mu)$ innerhalb der Grenzen $1-90 \%$.

Ausgeführte Berechnung ergab für das Volumgewicht $(\mathrm{Y})$ in linearer Abhängigkeit vom Kohlenstoffgehalt $\left(x_{1}\right)$, vom Tongehalt $\left(x_{2}\right)$ und vom Sandgehalt $\left(x_{4}\right)$ folgende Regressior sgleichung:

$$
\begin{aligned}
& \mathrm{Y}=1.40-0.072 \mathrm{x}_{1}-0.0013 \mathrm{x}_{2}+0.0014 \mathrm{x}_{4} \\
& \text { t-Werte der Koeffizienten: }
\end{aligned}
$$

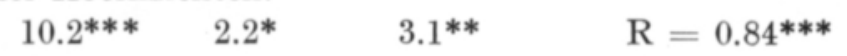

Neben dem selbstverständlichen Einfluss des Humusgehalts lässt die Gleichung erkennen, dass die Sandfraktion in der Tat einen deutlich das Volumgewicht erhöhenden Effekt und die Tonfraktion ebenfalls einen statistisch signifikanten, erniedrigenden Einfluss hat. Numerisch haben beide Koeffizienten gleiche Grösse und die entsprechenderı Einflüsse heben sich somit in leichteren Tonböden auf, die ungefähr gleiche Mengen Ton und Sand enthalten.

In Abb. 2 ist ein Vergleich der berechneten und gefundenen Werte angestellt worden. Gegebene:weise kann die Übereinstimmung keine ausserordentlich strenge sein, da neben der Bodenzusammensetzung auch andere Faktoren von Einfluss auf die Struktur sind. Auf alle Fälle tritt die Abhängigkeit mit solcher Deutlichkeit zutage, dass volle Berechtigung vorliegt, den Begriff nnormales Volumgewicht der Bodenart" zu verwenden. Dass das vom Korrelationskoeffizienten angezeigte Er-

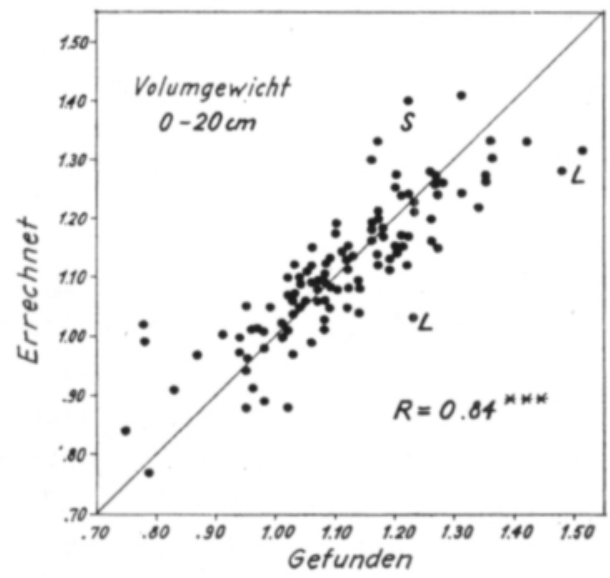

Abb. 2. Ubereinstimmung des gemessenen und des als Funktion der Bodenzusammensetzung berechneten Volumgewichts. 
klärungsvermögen nicht höher als dasjenige des in Abb. 1 dargestellten einfachen Modells ist, muss man in erster Linie auf den engeren Variationsbereich des Humusgehalts zurückführen.

Beim individuellen Betrachten solcher Einzelfälle, in denen sich eine bedeutende Abweichung zwischen dem berechneten und gefundenen Wert ergab, konnte u. a. folgendes beobachtet werden:

Die vier Punkte, neben denen in der Abbildung 2 der Buchstabe $\mathrm{S}$ angeschrieben ist, vertreten reine Sandböden, alle mit etwa $90 \%$ Sand $(>60 \mu)$. Die Variation innerhalb der Gruppe der unserem Material angehörenden Sandböden war gänzlich unabhängig vom Grobheitsgrad des Bodens; demnach scheint beim Überschreiten von $50 \%$ ein weiterer Anstieg der Sandfraktion das Volumengewicht der Ackerkrume nicht mehr zu erhöhen.

Mit L sind in der Abbildung drei aussergewöhnlich kompakte Lehmböden angezeigt. Die hohe Dichtigkeit trat in diesen Fällen auch u.a. in einer ungewöhnlich geringen nutzbaren Wasserkapazität zutage (2).

Die Böden mit Volumgewichten unter 1.00 sind grösstenteils humusreiche Tonböden. In diesem Bereich besteht eine erhebliche Streuung zwischen den be-

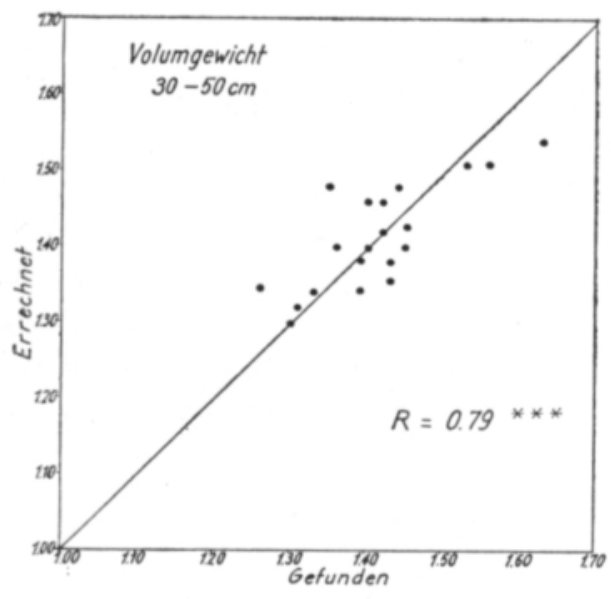

Abb. 3 .

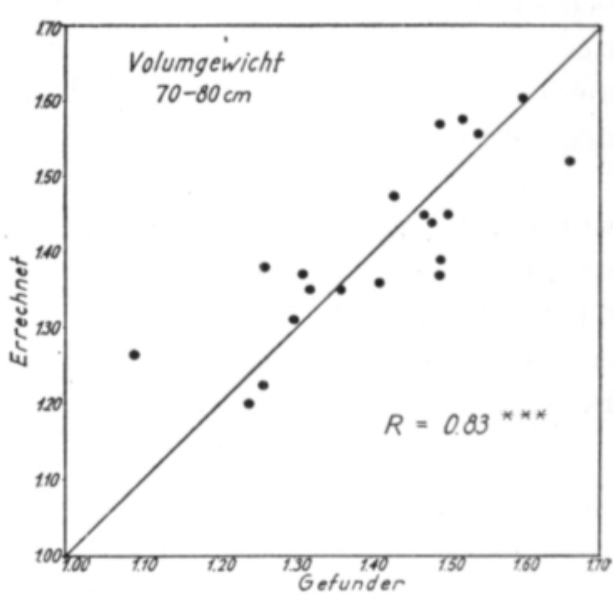

Abb. 4.

rechneten und gefundenen Werten. Als in einer zeitigeren Arbeit die stark humosen Tonböden als eigene Gruppe für sich untersucht wurden, zeigte es sich, dass deren Volumgewicht nicht nennenswert von der Bodenzusammensetzung abhängig war. Dagegen ging das Volumgewicht der stark humosen Tonböden mit zunehmender Menge der wasserstabilen Krümel stark herab. Das Volumgewicht der weniger stark humosen Tonböden ( $<4 \%$ org. C) stellte sich auch beim Untersuchen derselben als eigene Gruppe als Funktion der Zusammensetzung, ähnlich wie im gesamten, alle Bodenarten umfassenden Material, heraus. Überdies hatten aber die wasserstabilen Krümel eine leichte, das Volumgewicht erhöhende Wirkung (6). 
Der Verfasser hat zuvor eine Beschreibung der Porositätverhältnisse bei 28 Mineralbodenprofilen (30 bis $90 \mathrm{~cm}$ ) von wechselnder Textur veröffentlicht (4). Dieses Material wurde nun herangezogen, um zu ermitteln, in welchem Mass das Volumgewicht auch im Untergrund eine Funktion der Bodentextur ist. Die Sandböden sowie ein Schlicktonprofil mit 1.5\% organischem Material wurden aus der Betrachtung ausgeschlossen; für die übrigen 20 Profile wurde die das Volumgewicht (Y) in Abhängigkeit von den Mengen des Tons $<2 \mu\left(\mathrm{x}_{2}\right)$ und des Schluffs $2-20 \mu$ $\left(x_{3}\right)$ wiedergebende Funktion berechnet. Da die Veränderung des gesamten Hohlraumvolumens von der Oberfläche nach der Tiefe hin bei verschiedenen Bodenarten verschieden ist, wurden die Schichten $30-50 \mathrm{~cm}$ und $70-80 \mathrm{~cm}$ gesondert betrachtet. Auch die untere dieser Untersuchung unterzogene Schicht stand in sämtlichen Fällen deutlich über dem Grundwasserspiegel. So ergaben sich folgende Regressionsgleichungen:

$$
\begin{array}{ll}
30-10 \mathrm{~cm} & \\
\mathrm{Y}=1.42-0.0016 \mathrm{x}_{2}+0.0021 \mathrm{x}_{3} & \\
\mathrm{t}=1.84 \quad \mathrm{t}=1.70 & \mathrm{R}=0.79 * * * \\
70-80 \mathrm{~cm} & \\
\mathrm{Y}=1.58-0.0046 \mathrm{x}_{2}+0.0012 \mathrm{x}_{3} & \\
\mathrm{t}=5.00^{* * *} \mathrm{t}=0.92 & \mathrm{R}=0.83^{* * *}
\end{array}
$$

Die Ưbereinstimmung der berechneten und gefundenen Werte wird in Abb. 3 und 4 betrachtet. Man sieht, dass in beiden unteren Schichten auf Grund der Bodenzusammensetzung etwa 2/3 der Gesamtvariation des Volumgewichts, d.h. der gleiche Bruchteil wie auch bei der Ackerkrume erklärbar ist. Auch beim Untergrund kann man somit von dem (durch die Zusammensetzung bedingten) snormalen" Volumgewicht der Bodenarten sprechen.

Den Konstanten in Gl. (3) und (4) gemäss wäre bei grobkörnigen Böden (ohne abschlämmbare Teilchen) das Volumgewicht in $30-50 \mathrm{~cm}$ und $70-80 \mathrm{~cm}$ Tiefe 1.42 bzw. 1.58. Unter Annahme des spezifischen Gewichts 2.69 (5) wäre die entsprechende Gesamtporosität 47 bzw. $41 \%$. Nähere Betrachtung der aus der besprochenen Berechung ausgeschlossenen Sandböden zeigt, dass diese Zahlen der richtigen Grössenordnung s.nd.

Der Unterschied zwischen den auf den Ton bezogenen Regressionskoeffizienten in Gl. (3) bzw. (4) ist statistisch gesichert $\left(\mathrm{t}=2,36^{*}\right)$ und zeigt an, dass der herabsetzende Einfluss des Tons auf das Volumgewicht mit zunehmender Tiefe stärker wird. Übereinstirnmend hiermit kann man aus den die Hohlraumverhältnisse der Profile darstellenden Abbildungen (4) ersehen, dass in den schwersten Tonböden das gesamte Hohlraumvolumen von $30 \mathrm{~cm}$ Tiefe hinab zunimmt.

In bezug auf den Schluff ergab sich in Gl. (3) ein positiver Koeffizient von beträchtlicher Höne, dem allerdings nur die Wahrscheinlichkeit von $90 \%$ zukommt. Demgemäss würde der Schluff das Volumgewicht des oberen Untergrundes stärker 
erhöhen als die in den Berechnungen nicht berücksichtigten gröberen Fraktionen. Die niedrigste gemessene Gesamtporosität, 38-40\%, trat in der Tat bei dem reinsten Schluffprofil mit $60-70 \%$ Schluff $(2-20 \mu)$ auf.

\section{Uber die Anwendung des Volumgewichts in Untersuchungen}

Als Beispiel der Anwendung des Volumgewichts der Ackerkrume sei erwähnt, dass in eben den hier vorliegenden Material das Volumgewicht eine stark negative Korrelation mit der nutzbaren Wasserkapazität des Bodens bei allen solchen Bodenarten aufweist, die eine Neigung zur Krümelstruktur haben. In Sand- und Schluffböden zeigte sich keine solche Korrelation (2).

Oft wäre es auch notwendig, die Luftkapazität zu kennen, die gewöhnlicherweise als Differenz des gesamten Hohlraumvolumens und der Feldkapazität berechnet wird. Dazu müssen die Proben entnommen werden, wenn sich der Boden in Feldkapazitätzustand befindet, was auch im übrigen beispielsweise zum Ausschalten des Schrumpfungseinfluss und zum Herabsetzen der Saisonvariation empfehlenswert ist (8).

Zum Berechnen der Totalporosität muss ausser dem Volumgewicht das spezifische Gewicht des Bodens bekannt sein. Bei den finnischen Böden, die hinsichtlich ihrer Mineralzusammensetzung und Geologie verhältnismässig einheitlich sind, ist das spezifische Gewicht eine Funktion des Humusgehalts und der Textur $(5,7)$ Es kann mit einer für Porositätsuntersuchungen hinreichenden Genauigkeit aus folgender Formel berechnet werden:

$$
\text { Spez. Gew. }=2.69-0.042 \text { (org. total-C) }+0.001 \text { (Tongehalt) }
$$

\section{Zus a m m e nfassung}

Von der Gesamtvariation des Volumgewichts sowohl der Ackerkrume als auch des Untergrundes über dem Grundwasserspiegel sind etwa 2/3 auf Grund der Variationen des Humusgehalts und der Textur erklärbar. Dieses Resultat ergab sich aus einer Berechnung des Volumgewichts bei einer die glazialen Böden in SüdFinnland vertretenden Probenreihe in Abhängigkeit von den obengenannten Faktoren (Gl. 2 bis 4). Den von den Regressionsgleichungen angegebenen Wert hat der Verfasser als "normales Volumgewicht" bezeichnet, und dasselbe kann in verschiedenen Untersuchungen als Vergleichsgrundlage beim Beurteilen der Dichtigkeit des Bodens Verwendung finden.

Das Volumgewicht der Ackerkrume bestimmt sich in erster Linie nach dem Humusgehalt; daneben kann man aber einen das Volumgewicht herabsetzenden bzw. erhöhenden Einfluss der Tonfraktion bzw. der Sandfraktion wahrnehmen. Nach grösserer Tiefe hin wird der negative Einfluss der Tonfraktion stärker. 


\section{LITTERATURVERZEICHNIS}

(1) Frese, H. 1959. Netue Gesichtspunkte für die Beurteilung unserer Ackerböden. Landw. Forsch. Sonderh. 12: $49-59$.

(2) Heinonen, R. 1954 Multakerroksen kosteussuhteista Suomen maalajeissa. Summary: Moisture conditions in Finnish topsoils. Agrogeol. julk. 62: 1-82.

(3) - - 1955. Soil aggregation in relation to texture and organic matter. Ibid. 64: 1-17.

(4) - 1957. Pchjamaan huokoisuussuhteista Suomen maalajeissa. Summary: Porosity conditions in Finnish subsoils. J. Sci. Agr. Soc. Finland. 29: 27-37.

(5) - 1957. Sucmen maalajien ominaispainosta. Summary: On the specific gravity of Finnish soils. Ibid 29: $38-40$.

(6) - - 1958. Die Bedeutung des Humusgehaltes im Boden für die Krümelbildung. Deutsche Akad. Landw.wiss. Berlin, Tagungsber. 13: 279-285.

(7) - 1960. Der sity, amount, and carbon content of organic matter in soils - some methodical reflections J. Sci. Agr. Soc. Finland (Im Druck).

(8) Heinonen, R. \& FukкацA, R. 1954. Seasonal variation in soil structure. Ibid. 26: 116-119.

(9) Vomocil, J. A. 1957. Measurement of soil bulk density and penetrability: a review of methods. Adv. Agr. 9: 159-175.

\section{S E L O S T S :}

\section{TILAVUUSPAINO MAAN ,NORMAALIN, RAKENTEEN TUNNUKSENA}

\section{REIJO HeINONEN}

\section{Helsingin Yliopiston maanviljelyskemian laitos}

Etelä-Suomen vil elysmaita edustavasta aineistosta laskettiin maan tilavuuspaino humuspitoisuuden ja tekstuurin fınktiona. Saadut osittaisregressioyhtälöt (2-4) selittivät sekä multakerroksen että pohjamaan tilavuuspainon kokonaisvaihtelusta n. 2/3. Regressioyhtälöiden antamaa tulosta tekijä on nimittänyt maalajin snormaaliksi tilavuuspainoksi, ja sitä voidaan käyttää erilaisissa tutkimuksissa vertailuperustana maar. tiiviyttä arvosteltaessa.

Multakerroksen tilavuuspaino määräytyy ensi sijassa humuspitoisuuden mukaan, mutta sen ohella voidaan todeta saveks sn tilavuuspainoa alentava ja hiekan sitä kohottava vaikutus. Syvemmälle siirryttäessä saveksen regatiivinen vaikutus kasvaa. 